\title{
The second order perturbation approach for elliptic partial differential equations on random domains ${ }^{\text {th }}$
}

\author{
Helmut Harbrecht, Michael D. Peters \\ University of Basel, Department of Mathematics and Computer Science, Spiegelgasse 1, \\ CH-4051 Basel \\ \{helmut.harbrecht,michael.peters\}@unibas.ch
}

\begin{abstract}
The present article is dedicated to the solution of elliptic boundary value problems on random domains. We apply a high-precision second order shape Taylor expansion to quantify the impact of the random perturbation on the solution. Thus, we obtain a representation of the solution with third order accuracy in the size of the perturbation's amplitude. The major advantage of this approach is that we end up with purely deterministic equations for the solution's moments. In particular, we derive representations for the first four moments, i.e., expectation, variance, skewness and kurtosis. These moments are efficiently computable by means of boundary integral equations. Numerical results are presented to validate the presented approach.
\end{abstract}

Keywords: Boundary value problem; random domain; perturbation method.

\section{Introduction}

Often, practical problems from science and engineering result in the task of solving a boundary value problem for an unknown function. The numerical solution of such boundary value problems is in general well understood, at least if the problem's input parameters are known exactly. Often, however, the input parameters are not known exactly. Hence, the challenge is to obtain high-precision approximations also in the presence of uncertainties. Typically, random input parameters are then modelled in terms of random fields and, as a consequence, the given boundary value problem is turned

\footnotetext{
This research has been supported by the Swiss National Science Foundation (SNSF) through the project " $\mathcal{H}$-matrix based first and second moment analysis".
} 
into a random one. This yields a solution which is a random field itself. In this article, to keep the presentation simple for the reader's convenience, we shall consider the Dirichlet problem for the Poisson equation which is formulated relative to a random domain:

$$
-\Delta u(\omega)=f \text { in } D(\omega), \quad u(\omega)=g \text { on } \partial D(\omega) .
$$

Here, $D(\omega)$ denotes the domain under consideration with boundary $\partial D(\omega)$ which both depend on the random parameter $\omega \in \Omega$. Of course this problem can be easily extended for the case of more complex data, as for example a more complex diffusion coefficient, cf. [16]. The idea of taking random computational domains into account is inspired by tolerances in the fabrication process of a mechanical device or by damages of the boundary which appear during the life cycle of a device. Typically, such devices are close to a nominal shape but differ of course from its mathematical definition. Since these tolerances are in general small, we can also make the crucial assumption of the smallness of the random perturbations. Uncertainty quantification for computational domains arouses recently more interest, see $[4,13,14,17,25,30]$.

By identifying domains with their boundary, a random domain $D(\omega)$, which is close to a given nominal domain $D_{0}$, can be described as a normal perturbation of this nominal boundary $\partial D_{0}$ :

$$
\partial D(\omega)=\left\{\mathbf{y} \in \mathbb{R}^{n}: \mathbf{y}(\mathbf{x})=\mathbf{x}+\varphi(\mathbf{x}, \omega) \mathbf{n}(\mathbf{x}), \mathbf{x} \in \partial D_{0}\right\} .
$$

In this context, the random field $\varphi(\omega): \partial D_{0} \rightarrow \mathbb{R}$ is a scalar function which is defined with respect to the nominal boundary $\partial D_{0}$. It uniquely determines the domain perturbation via $\varphi(\omega) \mathbf{n}: \partial D_{0} \rightarrow \mathbb{R}^{n}$, with $\mathbf{n}$ denoting the outward normal to the domain $D_{0}$.

The most simple methodology to deal with randomness in numerical computations is the Monte-Carlo method, cf. [22, 28]. Here, numerous draws of the random input data are sampled according to some a-priorily known or empirical distribution. Each draw entails the computation of a deterministic boundary value problem. Then, the statistics like the mean and the variance of these samples are formed. Nevertheless, for boundary value problems on random domains, each sample implies a new domain and thus a new mesh, the assembly of new mass and stiffness matrices, etc. Therefore, the MonteCarlo method is extremely costly and rather difficult to implement for the problem at hand. Note that the same accounts for other more sophisticated quadrature techniques like the quasi-Monte Carlo quadrature, cf. [3], or sparse quadrature methods, cf. [2].

Thus, we aim here at a different approach, namely the perturbation approach, see $[1,13,14,17,20,21]$. It facilitates to approximate the random 
solution on an arbitrary compactum inside the fixed nominal domain $D_{0}$. The pivotal idea of the perturbation approach for random boundary value problems is the expansion of the underlying random field around the related input parameter's expectation, in our case the domain $D_{0}$, via a (shape-) Taylor expansion. For the boundary value problem (1) at hand, this will involve shape calculus, cf. [7, 26, 29]. With the help of the shape Taylor expansion, we can derive asymptotic expansions of the random output's expectation, variance and also higher order moments.

More precisely, we employ a second order shape Taylor expansion and derive corresponding asymptotic expansions for the first four moments. These can be computed explicitly under the finite noise assumption. This means, the random domain perturbation in (2) is of the form

$$
\varphi(\mathbf{x}, \omega)=\sum_{i=1}^{N} \varphi_{i}(\mathbf{x}) Y_{i}(\omega)
$$

with centered random variables $Y_{i}: \Omega \rightarrow[-1,1]$ which are independent and identically distributed, see $[5,30]$.

As we will show, in the setting (3), i.e., having $N$ terms in the series expansion of the random perturbation field $\varphi \mathbf{n}$, the expectation and the variance can be computed with a computational cost of order $\mathcal{O}(N)$. The skewness and kurtosis can be computed with a computational cost of order $\mathcal{O}\left(N^{2}\right)$.

We remark that similar approach for scalar output functionals of partial differential equations on uncertain domains has already been considered in [6]. Such shape functionals can be linearized by means of shape calculus, which, in particular, involves the computation of the shape Hessian of the functional under consideration. However, employing the adjoint method, which is well-known in shape optimization, only the first order shape derivative of the partial differential equation under consideration has to be computed. Whereas, for the problem considered in the present article, also the second order shape derivative of the partial differential equation has to be computed. The latter is computationally much more demanding.

The rest of this article is organized as follows. In Section 2, we introduce the basic ideas of shape calculus and derive the asymptotic expansions for the random solution's statistics. Then, in Section 3, we propose a way to compute these expansions by means of a boundary element method. Numerical results are presented in Section 4. Finally, we state concluding remarks in Section 5 


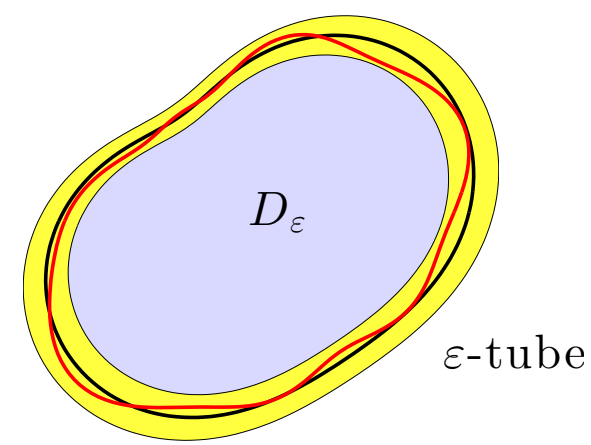

Figure 1: Illustration of the perturbed domain $D_{\varepsilon}$ (red boundary), the nominal domain $D_{0}$ (black boundary), and the $\varepsilon$-tube around $\partial D_{0}$ (yellow).

\section{Perturbation analysis}

To avoid the extreme high-dimensionality of a direct discretization of (1) by means of the domain mapping method, see e.g. [30], which is driven by the size $N$ of the expansion (3), a technique can be applied which is mainly known from shape sensitivity analysis, namely the so-called local shape derivative, see $[8,27]$. It has been established as a measure of the solution's dependence on domain or boundary perturbations. Such shape derivatives are in principle known since Hadamard, cf. [12] and nowadays well established in shape optimization, see [7, 18, 26, 29]. Since the solution's nonlinear dependence on the shape of the domain is Fréchet differentiable, we can linearize it around the nominal domain $D_{0}$. Thus, deterministic expressions for the solution's statistics can be derived.

\subsection{Shape calculus}

Consider a sufficiently smooth domain $D_{0}$ and a boundary variation in the direction of the outward normal $\mathbf{n}$ :

$$
\varphi \mathbf{n}: \partial D_{0} \rightarrow \mathbb{R}^{n} \quad \text { such that }\|\varphi\|_{C^{2,1}\left(\partial D_{0}\right)} \leq 1
$$

Then, the perturbed domain $D_{\varepsilon}$ can be defined as the interior of its boundary

$$
\partial D_{\varepsilon}=\left\{\mathbf{y} \in \mathbb{R}^{n}: \mathbf{y}(\mathbf{x})=\mathbf{x}+\varepsilon \varphi(\mathbf{x}) \mathbf{n}(\mathbf{x}), \mathbf{x} \in \partial D_{0}\right\} .
$$

The perturbed domain $D_{\varepsilon}$ is well defined for sufficiently small $\varepsilon>0$, see Figure 1 for a visualization of this situation. 
We intend to linearize the solution $u_{\varepsilon}$ of the boundary value problem on the perturbed domain

$$
-\Delta u_{\varepsilon}=f \text { in } D_{\varepsilon}, \quad u_{\varepsilon}=g \text { on } \partial D_{\varepsilon},
$$

around the nominal domain $D_{0}$. To that end, we assume throughout this article that the data $f, g: \mathbb{R}^{n} \rightarrow \mathbb{R}$ are sufficiently smooth in order to guarantee that the solution $u_{\varepsilon}$ is always contained in $C^{2}\left(\overline{D_{\varepsilon}}\right)$. This can be guaranteed if there holds $f \in C\left(\mathbb{R}^{n}\right)$ and $g \in C\left(\mathbb{R}^{n}\right)$, respectively cf. [10].

Under our smoothness assumptions, we can expand $u_{\varepsilon}$, for $\varepsilon>0$ sufficiently small, in a shape Taylor expansion by using first and second order local shape derivatives:

$$
u_{\varepsilon}(\mathbf{x})=u_{0}(\mathbf{x})+\varepsilon \delta u_{0}[\varphi](\mathbf{x})+\frac{\varepsilon^{2}}{2} \delta^{2} u_{0}[\varphi, \varphi](\mathbf{x})+\mathcal{O}\left(\varepsilon^{3}\right), \quad \mathbf{x} \in K \Subset D_{0} .
$$

Here, $K$ is an arbitrary compact subset of $D_{0}$, cf. $[8,9,27]$, and $u_{0}$ is the solution of the boundary value problem on the nominal domain

$$
-\Delta u_{0}=f \text { in } D_{0}, \quad u_{0}=g \text { on } \partial D_{0} .
$$

Moreover, the function $\delta u_{0}[\varphi]$ denotes the first order local shape derivative, which is given by

$$
\begin{aligned}
\Delta \delta u_{0}[\varphi] & =0 & & \text { in } D_{0}, \\
\delta u_{0}[\varphi] & =\varphi \frac{\partial\left(g-u_{0}\right)}{\partial \mathbf{n}} & & \text { on } \partial D_{0},
\end{aligned}
$$

and $\delta^{2} u_{0}\left[\varphi, \varphi^{\prime}\right]$ is the second order local shape derivative. The latter can be computed according to

$$
\begin{aligned}
\Delta \delta^{2} u_{0}\left[\varphi, \varphi^{\prime}\right] & =0 & \text { in } D_{0}, \\
\delta^{2} u_{0}\left[\varphi, \varphi^{\prime}\right] & =\varphi \varphi^{\prime} \frac{\partial^{2}\left(g-u_{0}\right)}{\partial \mathbf{n}^{2}}-\varphi \frac{\partial \delta u_{0}\left[\varphi^{\prime}\right]}{\partial \mathbf{n}}-\varphi^{\prime} \frac{\partial \delta u_{0}[\varphi]}{\partial \mathbf{n}} & \text { on } \partial D_{0}
\end{aligned}
$$

Notice that the convergence in (4) is uniform with respect to $L^{\infty}(K)$ as $\varepsilon \rightarrow 0$ provided that the set $K$ is fixed and contained in $D_{\varepsilon}$ for every sufficiently small $\varepsilon>0$.

\subsection{Random domains}

So far, we have introduced shape calculus to describe the solution's dependence on the domain's shape. In this section, we extend this approach towards random boundary variations. To that end, let $(\Omega, \mathcal{F}, \mathbb{P})$ denote 
a complete probability space, where $\Omega$ is the set of outcomes, $\mathcal{F}$ is a $\sigma$ algebra containing the possible events and $\mathbb{P}$ is a probability measure which describes the likeliness of each event, i.e., $\mathbb{P}: \mathcal{F} \rightarrow[0,1]$. In this context, complete means that $\mathcal{F}$ contains all $\mathbb{P}$-null sets. Then, we define the random perturbation field

$$
\varphi(\omega) \mathbf{n}: \partial D_{0} \rightarrow \mathbb{R}^{n}
$$

such that $\|\varphi(\omega)\|_{C^{2,1}\left(\partial D_{0}\right)} \leq 1 \mathbb{P}$-almost surely. For sufficiently small $\varepsilon>0$, we thus arrive at the random domain $D_{\varepsilon}(\omega)$ which is given via its boundary

$$
\partial D_{\varepsilon}(\omega)=\left\{\mathbf{y} \in \mathbb{R}^{n}: \mathbf{y}(\mathbf{x})=\mathbf{x}+\varepsilon \varphi(\mathbf{x}, \omega) \mathbf{n}(\mathbf{x}), \mathbf{x} \in \partial D_{0}\right\} .
$$

In view of the first and second order local shape derivatives, the solution $u_{\varepsilon}(\omega)$ of the random boundary value problem

$$
-\Delta u_{\varepsilon}(\omega)=f \text { in } D_{\varepsilon}(\omega), \quad u_{\varepsilon}(\omega)=g \text { on } \partial D_{\varepsilon}(\omega),
$$

can be expanded by the random shape Taylor expansion. For notational convenience, we introduce the abbreviations

$$
\delta u_{0}(\mathbf{x}, \omega):=\delta u_{0}[\varphi(\omega)](\mathbf{x}) \quad \text { and } \quad \delta^{2} u_{0}(\mathbf{x}, \omega):=\delta^{2} u_{0}[\varphi(\omega), \varphi(\omega)](\mathbf{x}) .
$$

It holds

$$
u_{\varepsilon}(\mathbf{x}, \omega)=u_{0}(\mathbf{x})+\varepsilon \delta u_{0}(\mathbf{x}, \omega)+\frac{\varepsilon^{2}}{2} \delta^{2} u_{0}(\mathbf{x}, \omega)+\mathcal{O}\left(\varepsilon^{3}\right), \quad \mathbf{x} \in K \Subset D_{0} .
$$

A visualization of the random domain with the inscribed compactum $K$ of evaluation is found in Figure 2. We arrive at the following theorem which is an extension of the corresponding result for the first order shape Taylor expansion, see [17].

Theorem 2.1. Assume that the random boundary perturbation $\varphi$ is centered, i.e. $\mathbb{E}[\varphi](\mathbf{x})=0$ for all $\mathbf{x} \in \partial D_{0}$. Then, there exists $\varepsilon_{0}>0$ such that for all $\varepsilon \leq \varepsilon_{0}$ and for all $\mathbf{x} \in K \Subset D_{0}$, the expectation $\mathbb{E}\left[u_{\varepsilon}\right]$ and the variance $\mathbb{V}\left[u_{\varepsilon}\right]$ admit the asymptotic expansions

$$
\begin{aligned}
& \mathbb{E}\left[u_{\varepsilon}\right](\mathbf{x})=u_{0}(\mathbf{x})+\frac{\varepsilon^{2}}{2} \mathbb{E}\left[\delta^{2} u_{0}\right](\mathbf{x})+\mathcal{O}\left(\varepsilon^{3}\right), \\
& \mathbb{V}\left[u_{\varepsilon}\right](\mathbf{x})=\varepsilon^{2} \mathbb{E}\left[\left(\delta u_{0}\right)^{2}\right](\mathbf{x})+\varepsilon^{3} \mathbb{E}\left[\delta u_{0} \delta^{2} u_{0}\right](\mathbf{x})+\mathcal{O}\left(\varepsilon^{4}\right) .
\end{aligned}
$$

Proof. Using the fact that $\mathbb{E}\left[\delta u_{0}\right](\mathbf{x})=0$ since $\varphi(\omega)$ is centered, the first equation follows immediately from taking the expectation on both sides of 


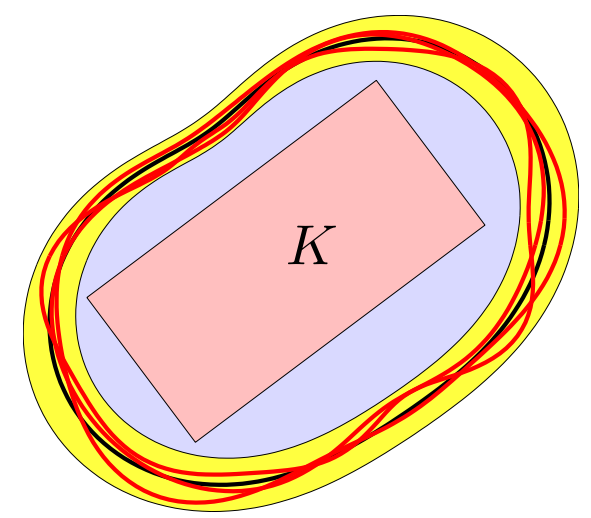

Figure 2: Illustration of the random domain with evaluation region $K$.

the shape Taylor expansion (9), cf. $[15,17]$. The second equation is obtained in accordance with

$$
\begin{aligned}
\mathbb{V}\left[u_{\varepsilon}\right] & =\mathbb{E}\left[\left(u_{\varepsilon}-\mathbb{E}\left[u_{\varepsilon}\right]\right)^{2}\right] \\
& =\mathbb{E}\left[\left(u_{0}+\varepsilon \delta u_{0}+\frac{\varepsilon^{2}}{2} \delta^{2} u_{0}-u_{0}-\frac{\varepsilon^{2}}{2} \mathbb{E}\left[\delta^{2} u_{0}\right]+\mathcal{O}\left(\varepsilon^{3}\right)\right)^{2}\right] \\
& =\varepsilon^{2} \mathbb{E}\left[\left(\delta u_{0}+\frac{\varepsilon}{2} \delta^{2} u_{0}-\frac{\varepsilon}{2} \mathbb{E}\left[\delta^{2} u_{0}\right]\right)^{2}\right]+\mathcal{O}\left(\varepsilon^{4}\right) \\
& =\varepsilon^{2} \mathbb{E}\left[\left(\delta u_{0}\right)^{2}\right]+\varepsilon^{3} \mathbb{E}\left[\delta u_{0} \delta^{2} u_{0}\right]+\mathcal{O}\left(\varepsilon^{4}\right),
\end{aligned}
$$

where we again use that $\mathbb{E}\left[\delta u_{0}\right](\mathbf{x})=0$, implying that

$$
\mathbb{E}\left[\delta u_{0} \mathbb{E}\left[\delta^{2} u_{0}\right]\right]=\mathbb{E}\left[\delta u_{0}\right] \mathbb{E}\left[\delta^{2} u_{0}\right]=0 .
$$

This theorem provides asymptotic expansions of the first two moments which are third and fourth order accurate in the perturbation size $\varepsilon$, respectively. In complete analogy, one can compute asymptotic expansions of the third and fourth moment (and even higher order moments if required).

Corollary 2.2. The third centered moment is given by

$$
\begin{aligned}
\mathbb{M}_{3}\left[u_{\varepsilon}\right](\mathbf{x})= & \mathbb{E}\left[\left(u_{\varepsilon}(\mathbf{x})-\mathbb{E}\left[u_{0}\right](\mathbf{x})\right)^{3}\right]=\varepsilon^{3} \mathbb{E}\left[\left(\delta u_{0}(\mathbf{x})\right)^{3}\right] \\
& +\frac{3 \varepsilon^{4}}{2} \mathbb{E}\left[\left(\delta u_{0}(\mathbf{x})\right)^{2}\left(\delta^{2} u_{0}(\mathbf{x})-\mathbb{E}\left[\delta^{2} u_{0}(\mathbf{x})\right]\right)\right]+\mathcal{O}\left(\varepsilon^{5}\right)
\end{aligned}
$$


and the fourth centered moment is given by

$$
\begin{aligned}
\mathbb{M}_{4}\left[u_{\varepsilon}\right](\mathbf{x})= & \mathbb{E}\left[\left(u_{\varepsilon}(\mathbf{x})-\mathbb{E}\left[u_{0}\right](\mathbf{x})\right)^{4}\right]=\varepsilon^{4} \mathbb{E}\left[\left(\delta u_{0}(\mathbf{x})\right)^{4}\right] \\
& +2 \varepsilon^{5} \mathbb{E}\left[\left(\delta u_{0}(\mathbf{x})\right)^{3}\left(\delta^{2} u_{0}(\mathbf{x})-\mathbb{E}\left[\delta^{2} u_{0}(\mathbf{x})\right]\right)\right]+\mathcal{O}\left(\varepsilon^{6}\right) .
\end{aligned}
$$

\subsection{Computation of the asymptotic expansions}

Let us assume that the random field is given in form of the expansion

$$
\varphi(\mathbf{x}, \omega)=\sum_{i=1}^{N} \varphi_{i}(\mathbf{x}) Y_{i}(\omega)
$$

where the random variables $Y_{i}: \Omega \rightarrow[-1,1]$ are centered, independent and identically distributed and where the spatial coefficient functions $\left\{\varphi_{i}\right\}_{i}$ form a subset of $C^{2,1}\left(\partial D_{0}\right)$. Notice that, if $\varphi(\mathbf{x}, \omega)$ is not centered, we can redefine the reference domain $D_{0}$ as the interior of the boundary

$$
\partial \widetilde{D}_{0}=\left\{\mathbf{y} \in \mathbb{R}^{n}: \mathbf{y}(\mathbf{x})=\mathbf{x}+\mathbb{E}[\varphi](\mathbf{x}) \mathbf{n}(\mathbf{x}), \mathbf{x} \in \partial D_{0}\right\} .
$$

The random perturbations are then centered with respect to the transformed reference domain $\widetilde{D}_{0}$.

Lemma 2.3. Let $\varphi(\mathbf{x}, \omega)$ be given by (13). Then, there holds

$$
\delta u_{0}[\varphi(\mathbf{x}, \omega)]=\sum_{i=1}^{N} \delta u_{0}\left[\varphi_{i}\right](\mathbf{x}) Y_{i}(\omega)
$$

and

$$
\delta^{2} u_{0}[\varphi(\mathbf{x}, \omega), \varphi(\mathbf{x}, \omega)]=\sum_{i, j=1}^{N} \delta^{2} u_{0}\left[\varphi_{i}, \varphi_{j}\right](\mathbf{x}) Y_{i}(\omega) Y_{j}(\omega) .
$$

Proof. The claim for the first order local shape derivative follows directly from the linearity of the first order local shape derivative with the representation (13). For the second order local shape derivative, the assertion is obtained from its bilinearity, again, together with the representation (13) for the random field. 
With this lemma at hand, together with the multinomial theorem where $\boldsymbol{\alpha}=\left(\alpha_{1}, \ldots, \alpha_{N}\right)$ denotes an $N$-dimensional multiindex, we derive

$$
\begin{aligned}
\mathbb{E}\left[\left(\delta u_{0}\right)^{k}\right](\mathbf{x}) & =\mathbb{E}\left[\left(\sum_{i=1}^{N} \delta u_{0}\left[\varphi_{i}\right](\mathbf{x}) Y_{i}\right)^{k}\right] \\
& =\mathbb{E}\left[\sum_{|\boldsymbol{\alpha}|=k}\left(\begin{array}{l}
k \\
\boldsymbol{\alpha}
\end{array}\right) \prod_{i=1}^{N}\left(\delta u_{0}\left[\varphi_{i}\right](\mathbf{x}) Y_{i}\right)^{\alpha_{i}}\right] \\
& =\sum_{|\boldsymbol{\alpha}|=k}\left(\begin{array}{l}
k \\
\boldsymbol{\alpha}
\end{array}\right) \prod_{i=1}^{N}\left(\delta u_{0}\left[\varphi_{i}\right](\mathbf{x})\right)^{\alpha_{i}} \mathbb{E}\left[\prod_{i=1}^{N} Y_{i}^{\alpha_{i}}\right] .
\end{aligned}
$$

In order to obtain expressions for the first order local shape derivative's moments up to order four, we discuss these cases now explicitly. As we will see, the expressions become more involved for increasing $k$ due to the increasing number of possible configurations for $|\boldsymbol{\alpha}|=k$.

- For $k=1$, we have only the situation $\left\{\alpha_{i}=1\right\}$ for one $i \in\{1, \ldots, N\}$ and thus, due to the centeredness of the $Y_{i}$, that

$$
\mathbb{E}\left[\prod_{i=1}^{N} Y_{i}^{\alpha_{i}}\right]=0
$$

- In the case $k=2$, we face the situations $\left\{\alpha_{i}=1, \alpha_{j}=1\right\}$ and $\left\{\alpha_{i}=2\right\}$ for distinct $i, j \in\{1, \ldots, N\}$. This results in

$$
\mathbb{E}\left[\prod_{i=1}^{N} Y_{i}^{\alpha_{i}}\right]=\mathbb{V}\left[Y_{i}\right] \quad \text { if } \alpha_{i}=2
$$

Otherwise, we end up with $\mathbb{E}\left[\prod_{i=1}^{N} Y_{i}^{\alpha_{i}}\right]=0$ by the independence of the random variables.

- For $k=3$, we have the situations $\left\{\alpha_{i}=1, \alpha_{j}=1, \alpha_{k}=1\right\},\left\{\alpha_{i}=\right.$ $\left.2, \alpha_{j}=1\right\}$ and $\left\{\alpha_{i}=3\right\}$ for distinct $i, j, k \in\{1, \ldots, N\}$. Again, the independence and the centeredness imply that $\mathbb{E}\left[\prod_{i=1}^{N} Y_{i}^{\alpha_{i}}\right]=0$ in the first two cases. In the third case, we obtain

$$
\mathbb{E}\left[\prod_{i=1}^{N} Y_{i}^{\alpha_{i}}\right]=\mathbb{E}\left[Y_{i}^{3}\right] \quad \text { if } \alpha_{i}=3 .
$$


- Finally, for $k=4$, we face the situations $\left\{\alpha_{i}=1, \alpha_{j}=1, \alpha_{k}=1, \alpha_{\ell}=\right.$ $1\},\left\{\alpha_{i}=2, \alpha_{j}=1, \alpha_{k}=1\right\},\left\{\alpha_{i}=2, \alpha_{j}=2\right\},\left\{\alpha_{i}=3, \alpha_{j}=1\right\}$ and $\left\{\alpha_{i}=4\right\}$ for distinct $i, j, k, \ell \in\{1, \ldots, N\}$. In cases one and three, the independence and the centeredness of the random variables imply that $\mathbb{E}\left[\prod_{i=1}^{N} Y_{i}^{\alpha_{i}}\right]=0$. Thus, we finally obtain

$$
\mathbb{E}\left[\prod_{i=1}^{N} Y_{i}^{\alpha_{i}}\right]= \begin{cases}\mathbb{V}\left[Y_{i}\right] \mathbb{V}\left[Y_{j}\right] & \text { if } \alpha_{i}=\alpha_{j}=2 \\ \mathbb{E}\left[Y_{i}^{4}\right] & \text { if } \alpha_{i}=4\end{cases}
$$

By combining these computations, we have just shown the following

Lemma 2.4. For random fields of the form (13), it holds

$$
\mathbb{E}\left[\left(\delta u_{0}\right)^{k}\right](\mathbf{x})= \begin{cases}0, & k=1, \\ \mathbb{V}[Y] \sum_{i=1}^{N}\left(\delta u_{0}\left[\varphi_{i}\right](\mathbf{x})\right)^{2}, & k=2, \\ \mathbb{E}\left[Y^{3}\right] \sum_{i=1}^{N}\left(\delta u_{0}\left[\varphi_{i}\right](\mathbf{x})\right)^{3}, & k=3, \\ \left(\mathbb{E}\left[Y^{4}\right]-3 \mathbb{V}[Y]^{2}\right) \sum_{i=1}^{N}\left(\delta u_{0}\left[\varphi_{i}\right](\mathbf{x})\right)^{4}+3\left(\mathbb{E}\left[\left(\delta u_{0}\right)^{2}\right](\mathbf{x})\right)^{2}, & k=4 .\end{cases}
$$

Notice that the aforementioned equations are also feasible to compute the terms

$$
\mathbb{E}\left[\left(\delta u_{0}\right)^{k} \mathbb{E}\left[\delta^{2} u_{0}\right]\right](\mathbf{x})=\mathbb{E}\left[\left(\delta u_{0}\right)^{k}\right](\mathbf{x}) \cdot \mathbb{E}\left[\delta^{2} u_{0}\right](\mathbf{x})
$$

for $k=2,3$ which appear in the asymptotic expansions (11) and (12) of the third and fourth centered moments.

Another term we shall provide is $\mathbb{E}\left[\left(\delta u_{0}\right)^{k} \delta^{2} u_{0}\right](\mathbf{x})$ for $k=0,1,2,3$. It can be computed in complete analogy by employing Lemma 2.3. We find

$$
\begin{aligned}
\mathbb{E} & {\left[\left(\delta u_{0}\right)^{k} \delta^{2} u_{0}\right](\mathbf{x}) } \\
& =\mathbb{E}\left[\sum_{|\boldsymbol{\alpha}|=k}\left(\begin{array}{l}
k \\
\boldsymbol{\alpha}
\end{array}\right) \prod_{\ell=1}^{N}\left(\delta u_{0}\left[\varphi_{\ell}\right](\mathbf{x}) Y_{\ell}\right)^{\alpha_{\ell}} \sum_{i, j=1}^{N} \delta^{2} u_{0}\left[\varphi_{i}, \varphi_{j}\right](\mathbf{x}) Y_{i} Y_{j}\right] \\
& =\sum_{|\boldsymbol{\alpha}|=k}\left(\begin{array}{l}
k \\
\boldsymbol{\alpha}
\end{array}\right) \prod_{\ell=1}^{N}\left(\delta u_{0}\left[\varphi_{\ell}\right](\mathbf{x})\right)^{\alpha_{\ell}} \sum_{i, j=1}^{N} \delta^{2} u_{0}\left[\varphi_{i}, \varphi_{j}\right](\mathbf{x}) \mathbb{E}\left[Y_{i} Y_{j} \prod_{\ell=1}^{N} Y_{\ell}^{\alpha_{\ell}}\right] .
\end{aligned}
$$


In view of the previous computations and

$$
|\boldsymbol{\alpha}|=5 \Longrightarrow \mathbb{E}\left[\prod_{i=1}^{N} Y_{i}^{\alpha_{i}}\right]= \begin{cases}\mathbb{V}\left[Y_{i}\right] \mathbb{E}\left[Y_{j}^{3}\right], & \text { if } \alpha_{i}=2, \alpha_{j}=3, \\ \mathbb{E}\left[Y_{i}^{3}\right] \mathbb{V}\left[Y_{j}\right], & \text { if } \alpha_{i}=3, \alpha_{j}=2, \\ \mathbb{E}\left[Y_{i}^{5}\right], & \text { if } \alpha_{i}=5, \\ 0, & \text { otherwise }\end{cases}
$$

straightforward calculation yields the subsequent result.

Lemma 2.5. For random fields of the form (13), it holds

$$
\begin{aligned}
& \mathbb{E}\left[\left(\delta u_{0}\right)^{k} \delta^{2} u_{0}\right](\mathbf{x})
\end{aligned}
$$

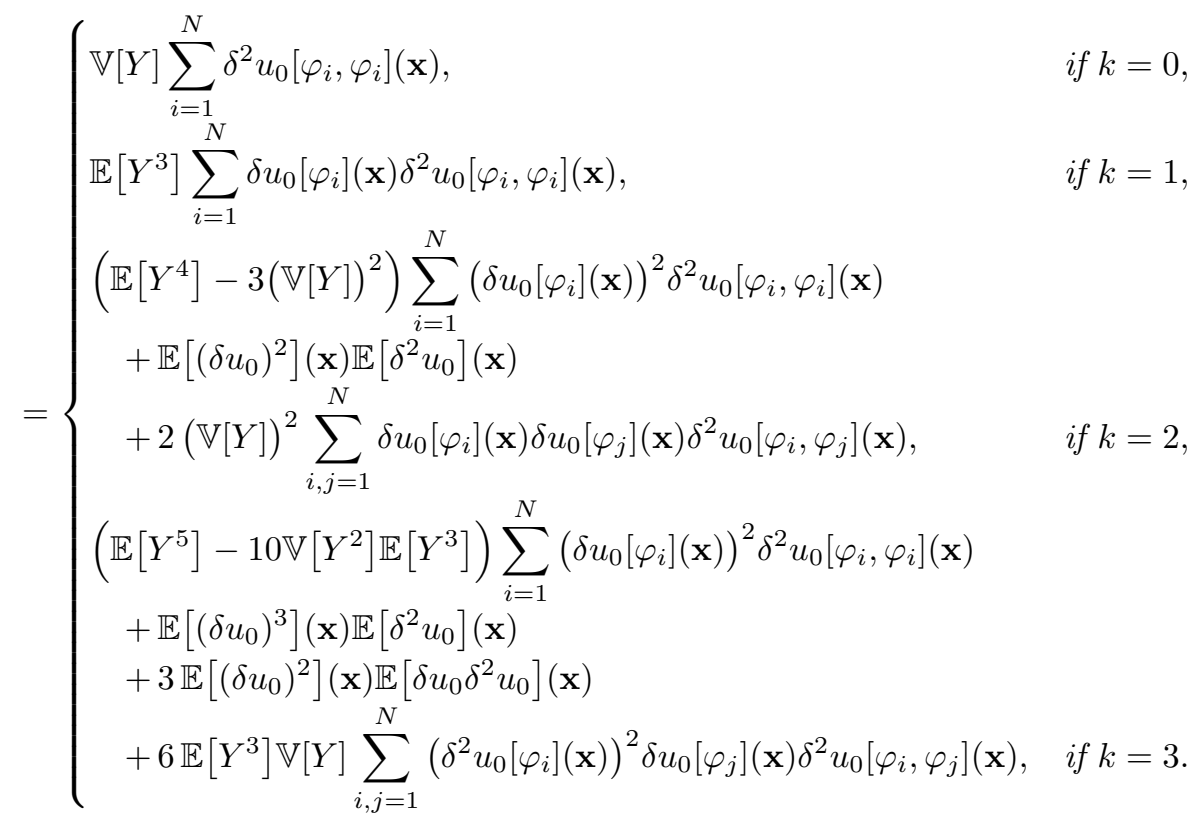

To summarize, we have provided by now easily computable expressions that are feasible to evaluate the asymptotic expansions for the expectation and the variance in accordance with (10), as well as the skewness (11) and the kurtosis (12). The computational cost is $\mathcal{O}(N)$ for the expectation and the variance while the computational cost is $\mathcal{O}\left(N^{2}\right)$ for the third and fourth centered moment.

Remark 2.6. If the law of $Y$ is symmetric, then it holds $\mathbb{E}\left[Y(\omega)^{3}\right]=0$ and $\mathbb{E}\left[Y(\omega)^{5}\right]=0$. Consequently, the asymptotic expansion of the variance simplifies in accordance with

$$
\mathbb{V}\left[u_{\varepsilon}\right](\mathbf{x})=\varepsilon^{2} \mathbb{E}\left[\left(\delta u_{0}(\mathbf{x}, \omega)\right)^{2}\right](\mathbf{x})+\mathcal{O}\left(\varepsilon^{4}\right), \quad \mathbf{x} \in K \Subset D_{0} .
$$


The same accounts for the fourth moment, which simplifies to

$$
\mathbb{M}_{4}\left[u_{\varepsilon}\right](\mathbf{x})=\varepsilon^{4} \mathbb{E}\left[\left(\delta u_{0}(\mathbf{x})\right)^{4}\right]+\mathcal{O}\left(\varepsilon^{6}\right) .
$$

See in particular (25) for a representation of all four moments in the case of the uniform distribution.

\section{Boundary integral equations}

In this section, we shall provide a means to compute the solution $u_{0}$ to the boundary value problem (5) and the associated local shape derivatives $\delta u_{0}[\varphi]$ and $\delta^{2} u_{0}[\varphi]$ given by (6) and (7), respectively. Since we only deal with boundary perturbations here, a natural approach is based on a boundary integral formulation, which circumvents the discretization of the entire domain. In particular, we shall explain how one may compute first and second order normal derivates of the solution $u_{0}$ to (5) by the boundary element method. For the sake of simplicity in the representation, we restrict ourselves from now on to the two-dimensional situation, i.e., $n=2$. Nevertheless, we emphasize that the three-dimensional situation can be treated in complete analogy.

\subsection{Newton potential}

In order to apply the boundary element method to the Poisson equation with non-homogenous loading, we make the ansatz

$$
u_{0}=v+N_{f}
$$

for a suitable Newton potential $N_{f}$ which satisfies the equation $-\Delta N_{f}=$ $f$ and a harmonic function $v$ which solves the associated boundary value problem with homogeneous right hand side

$$
\Delta v=0 \text { in } D_{0}, \quad v=g-N_{f} \text { on } \partial D_{0} .
$$

The Newton potential has to be either known analytically or it has to be computed in advance in a fairly simple domain $\widehat{D}$ which contains the domain $D_{0}$. To this end, efficient solution techniques for the Poisson equation, like higher-order finite elements and multigrid methods, can easily be applied, see [19]. 


\subsection{Dirichlet-to-Neumann map}

Now, in order to determine the solution $v$ of the Laplace equation (15), we employ a reformulation in terms of a boundary integral equation. Due to the knowledge of the Green's function of the underlying differential operator, we can easily represent the solution $v(\mathbf{x})$ of (15) at each point $\mathbf{x}$ inside the domain $D_{0}$ according to the representation formula

$$
v(\mathbf{x})=\int_{\partial D_{0}}\left\{G(\mathbf{x}, \mathbf{y}) \frac{\partial v}{\partial \mathbf{n}}(\mathbf{y})-\frac{\partial G(\mathbf{x}, \mathbf{y})}{\partial \mathbf{n}_{\mathbf{y}}} v(\mathbf{y})\right\} \mathrm{d} \sigma_{\mathbf{y}} .
$$

Therefore, the solution $v$ is uniquely determined from the knowledge of its Cauchy data $(v, \partial v / \partial \mathbf{n})$ on $\partial D_{0}$. Letting $\mathbf{x} \in D_{0}$ tend to the boundary $\partial D_{0}$ in (16) and taking into account the jump properties of the layer potentials, cf. [23], we obtain the direct boundary integral formulation of the problem:

$$
\frac{1}{2} v(\mathbf{x})=\int_{\partial D_{0}} G(\mathbf{x}, \mathbf{y}) \frac{\partial v}{\partial \mathbf{n}}(\mathbf{y}) \mathrm{d} \sigma_{\mathbf{y}}-\int_{\partial D_{0}} \frac{\partial G(\mathbf{x}, \mathbf{y})}{\partial \mathbf{n}_{\mathbf{y}}} v(\mathbf{y}) \mathrm{d} \sigma_{\mathbf{y}}, \quad \mathbf{x} \in \partial D_{0} .
$$

Next, we introduce the boundary integral operators which are associated

with the Laplace operator in two spatial dimensions. Since the fundamental solution is then given by

$$
G(\mathbf{x}, \mathbf{y})=-\frac{1}{2 \pi} \log \|\mathbf{x}-\mathbf{y}\|
$$

the single layer operator reads as

$$
\begin{aligned}
\mathcal{V}: H^{-1 / 2}\left(\partial D_{0}\right) & \rightarrow H^{1 / 2}\left(\partial D_{0}\right) \\
(\mathcal{V} \rho)(\mathbf{x}) & =-\frac{1}{2 \pi} \int_{\partial D_{0}} \log \|\mathbf{x}-\mathbf{y}\| \rho(\mathbf{y}) \mathrm{d} \sigma_{\mathbf{y}}
\end{aligned}
$$

while the double layer operator is given by

$$
\begin{aligned}
& \mathcal{K}: L^{2}\left(\partial D_{0}\right) \rightarrow L^{2}\left(\partial D_{0}\right), \\
&(\mathcal{K} \rho)(\mathbf{x})=\frac{1}{2 \pi} \int_{\partial D_{0}} \frac{\left\langle\mathbf{x}-\mathbf{y}, \mathbf{n}_{\mathbf{y}}\right\rangle}{\|\mathbf{x}-\mathbf{y}\|^{2}} \rho(\mathbf{y}) \mathrm{d} \sigma_{\mathbf{y}}
\end{aligned}
$$

Hence, by incorporating the Dirichlet boundary condition $u=g-N_{f}$ on $\partial D_{0}$, we can rewrite (17) in accordance with

$$
\mathcal{V} \frac{\partial v}{\partial \mathbf{n}}=\left(\frac{1}{2}+\mathcal{K}\right)\left(g-N_{f}\right) \quad \text { on } \partial D_{0} .
$$


This is the Dirichlet-to-Neumann map for the harmonic function $v$. It facilitates the computation of the missing Neumann data from the known Dirichlet data $g-N_{f}$ on $\partial D_{0}$. The single layer operator on the left hand side is symmetric and $H^{-1 / 2}\left(\partial D_{0}\right)$-elliptic provided that diam $\left(\partial D_{0}\right)<1$. In view of the Lax-Milgram lemma, this is sufficient to ensure the unique solvability of the boundary integral equation (20).

\subsection{Computing first and second order local shape derivates}

In complete analogy, the Neumann data of the local shape derivative $\delta u_{0}[\varphi]$ are easily computable by applying again the Dirichlet-to-Neumann. It holds

$$
\mathcal{V} \frac{\partial \delta u_{0}[\varphi]}{\partial \mathbf{n}}=\left(\frac{1}{2}+\mathcal{K}\right)\left(\varphi \frac{\partial\left(g-v-N_{f}\right)}{\partial \mathbf{n}}\right) \quad \text { on } \partial D_{0}
$$

Thus, similarly to (16), the local shape derivative at the point $\mathbf{x} \in D_{0}$ is given by

$\delta u_{0}[\varphi](\mathbf{x})=\int_{\partial D_{0}}\left\{G(\mathbf{x}, \mathbf{y}) \frac{\partial \delta u_{0}[\varphi]}{\partial \mathbf{n}}(\mathbf{y})-\frac{\partial G(\mathbf{x}, \mathbf{y})}{\partial \mathbf{n}_{\mathbf{y}}}\left(\varphi(\mathbf{y}) \frac{\partial\left(g-v-N_{f}\right)}{\partial \mathbf{n}}(\mathbf{y})\right)\right\} \mathrm{d} \sigma_{\mathbf{y}}$.

The computation of the second order local shape derivative $\delta^{2} u_{0}\left[\varphi, \varphi^{\prime}\right]$ is more sophisticated since its Dirichlet boundary data involve the term $\partial^{2} u_{0} / \partial \mathbf{n}^{2}$, cf. (7). The next lemma provides a formula that facilitates the computation of this term.

Lemma 3.1. There holds the identity

$$
\frac{\partial^{2} u_{0}}{\partial \mathbf{n}^{2}}=-\frac{\partial^{2} g}{\partial \mathbf{t}^{2}}-\kappa\left(\frac{\partial v}{\partial \mathbf{n}}-\frac{\partial\left(g-N_{f}\right)}{\partial \mathbf{n}}\right)-f
$$

where $\kappa$ denotes the curvature of $\partial D_{0}$.

Proof. Since $u_{0} \in C^{2}\left(\overline{D_{0}}\right)$, it holds the following identity for the LaplaceBeltrami operator $\Delta_{\Gamma}$ :

$$
-f=\Delta u_{0}=\frac{\partial^{2} u_{0}}{\partial \mathbf{n}^{2}}+\kappa \frac{\partial u_{0}}{\partial \mathbf{n}}+\Delta_{\Gamma} u_{0}
$$

In view of $u_{0}=v+N_{f}, \Delta_{\Gamma}\left(u_{0}-g\right)=0$ and

$$
\Delta_{\Gamma} g=\frac{\partial^{2} g}{\partial \mathbf{t}^{2}}-\kappa \frac{\partial g}{\partial \mathbf{n}},
$$

we obtain the assertion. 
For the computation of the random shape Tayler expansion (9) and thus also for the computation of the random solution's statistics, the second order local shape derivative has only to be known inside the set $K \Subset D_{0}$. Hence, we can use the indirect ansatz

$$
\delta^{2} u_{0}\left[\varphi, \varphi^{\prime}\right](\mathbf{x})=\int_{\partial D_{0}} G(\mathbf{x}, \mathbf{y}) \rho(\mathbf{y}) \mathrm{d} \sigma_{\mathbf{y}}, \quad \mathbf{x} \in D_{0}
$$

The unknown density $\rho \in H^{-1 / 2}\left(\partial D_{0}\right)$ is thus given by the first kind integral equation, see [23],

$$
\mathcal{V} \rho=\varphi \varphi^{\prime} \frac{\partial^{2}\left(g-u_{0}\right)}{\partial \mathbf{n}^{2}}-\varphi \frac{\partial \delta u_{0}\left[\varphi^{\prime}\right]}{\partial \mathbf{n}}-\varphi^{\prime} \frac{\partial \delta u_{0}[\varphi]}{\partial \mathbf{n}} \quad \text { on } \partial D_{0}
$$

\subsection{Solving boundary integral equations}

The next step towards the solution of the boundary value problem is the numerical approximation of the integral operators included in (20), (21) and (22), which involves a suitable parametrization of the occuring integrals. To that end, we introduce the parametrization $\gamma:[0,2 \pi] \rightarrow \partial D_{0}$ for the boundary $\partial D_{0}$. A very efficient numerical method for the approximation of the unknown Cauchy data is obtained by the collocation method when using trigonometric polynomials as ansatz functions. Then, the application of the trapezoidal rule for the numerical quadrature and an appropriate regularization to deal with the singular integrals yields a second order convergent method. If the data at hand and also the boundary are arbitrarily smooth, it is easy to show that this method is even exponentially convergent, cf. [23].

\section{Numerical results}

For the numerical examples, we consider the Poisson equation

$$
-\Delta u(\omega)=1 \text { in } D_{\varepsilon}(\omega), \quad u(\omega)=0 \text { on } \partial D_{\varepsilon}(\omega),
$$

on the randomly varying disc

$$
\begin{aligned}
D_{\varepsilon}(\omega) & =\left\{(\rho(\phi) \cos (\phi), \rho(\phi) \sin (\phi)) \in \mathbb{R}^{2}:\right. \\
0 & \left.\leq \rho(\phi)<r(\phi, \omega)=\frac{2}{5}+\frac{\varepsilon}{80} \sum_{\ell=1}^{5} Y_{2 \ell}(\omega) \cos (\ell \phi)+Y_{2 \ell-1}(\omega) \sin (\ell \phi)\right\}
\end{aligned}
$$


with $\varepsilon \leq 1 / 2$ and independent random variables $Y_{1}, \ldots, Y_{10} \sim \mathcal{U}([-1,1])$. The nominal domain $D_{0}$ is thus the disc of radius $2 / 5$ :

$$
D_{0}=\left\{(\rho(\phi) \cos (\phi), \rho(\phi) \sin (\phi)) \in \mathbb{R}^{2}: 0 \leq \rho(\phi)<\frac{2}{5}, 0 \leq \phi<2 \pi\right\} .
$$

A visualization of different realizations of the randomly varying domain in case of $\varepsilon=1 / 2$ is found in Figure 3 .

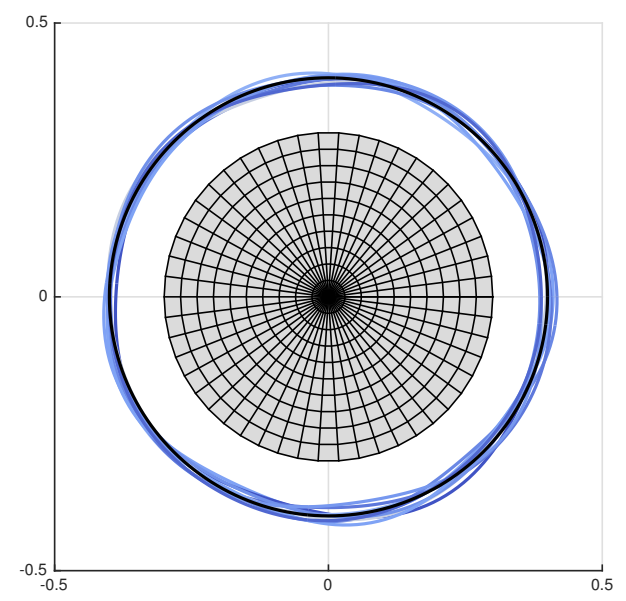

Figure 3: Different realizations (blue) of the boundary of $D_{1 / 2}(\omega)$ with inscribed nonvarying compactum $K$ (grey).

One readily verifies that the moments of the random variables satisfy

$$
\mathbb{E}\left[Y_{\ell}^{k}\right]= \begin{cases}0, & \text { if } k \text { odd } \\ \frac{1}{k+1}, & \text { if } k \text { even }\end{cases}
$$

Consequently, we obtain along the lines of Lemmata 2.4 and 2.5 the expansions

$$
\begin{aligned}
& \mathbb{E}\left[u_{\varepsilon}\right](\mathbf{x})=u_{0}(\mathbf{x})+\frac{\varepsilon^{2}}{6} \sum_{i=1}^{N} \delta^{2} u_{0}\left[\varphi_{i}, \varphi_{i}\right](\mathbf{x})+\mathcal{O}\left(\varepsilon^{3}\right), \\
& \mathbb{V}\left[u_{\varepsilon}\right](\mathbf{x})=\frac{\varepsilon^{2}}{6} \sum_{i=1}^{N}\left(\delta u_{0}\left[\varphi_{i}\right](\mathbf{x})\right)^{2}+\mathcal{O}\left(\varepsilon^{4}\right),
\end{aligned}
$$

for the expectation and variance. Whereas, for the skewness and the kurto- 
sis, we arrive at

$$
\begin{aligned}
& \mathbb{M}_{3}\left[u_{\varepsilon}\right](\mathbf{x})=\frac{3 \varepsilon^{4}}{2}[ \frac{2}{9} \sum_{i, j=1}^{N} \delta u_{0}\left[\varphi_{i}\right](\mathbf{x}) \delta u_{0}\left[\varphi_{j}\right](\mathbf{x}) \delta^{2} u_{0}\left[\varphi_{i}, \varphi_{j}\right](\mathbf{x}) \\
&\left.-\frac{2}{15} \sum_{i=1}^{N}\left(\delta u_{0}\left[\varphi_{i}\right](\mathbf{x})\right)^{2} \delta^{2} u_{0}\left[\varphi_{i}, \varphi_{i}\right](\mathbf{x})\right]+\mathcal{O}\left(\varepsilon^{5}\right), \\
& \mathbb{M}_{4}\left[u_{\varepsilon}\right](\mathbf{x})=\varepsilon^{4}\left[\frac{1}{3}\left(\sum_{i=1}^{N}\left(\delta u_{0}\left[\varphi_{i}\right](\mathbf{x})\right)^{2}\right)^{2}-\frac{2}{15} \sum_{i=1}^{N}\left(\delta u_{0}\left[\varphi_{i}\right](\mathbf{x})\right)^{4}\right]+\mathcal{O}\left(\varepsilon^{6}\right) .
\end{aligned}
$$

For varying $\varepsilon$, we will compare these asymptotic expansions with results derived by a sparse grid quadrature based on Clenshaw-Curtis quadrature points ${ }^{1}$, cf. [11]. To that end, we evaluate the samples on the compactum $K=\left\{\mathbf{x} \in \mathbb{R}^{2}:\|\mathbf{x}\|_{2} \leq 0.3\right\}$ in order to compute the solution's first four moments. Notice that, for all $\varepsilon \leq 0.5$, the compactum $K$ is almost surely contained in $D_{\varepsilon}(\omega)$, see also Figure 3 . In order to validate

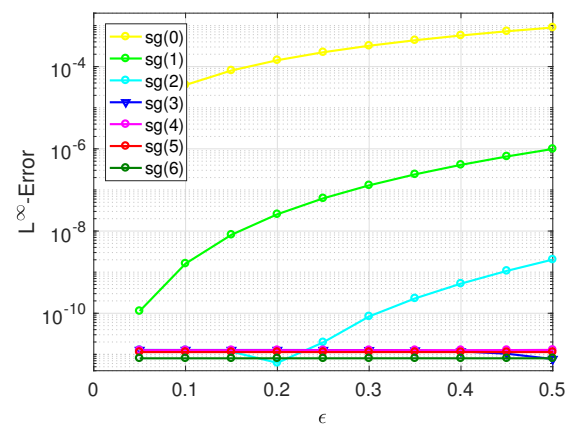

Figure 4: Error of the sparse grid quadrature for the mean.

the sparse grid quadrature, Figure 4 provides a convergence plot of the expectation for increasing numbers of quadrature points. We have $N(q)=$ $1,23,265,2069,12497,63097,280017$ quadrature points for $q=0,1, \ldots, 6$. As it turns out, from level $q=3$ on, we already have a very accurate approximation for the mean and expect a similar behavior for the higher order moments.

\footnotetext{
${ }^{1}$ The employed implementation of the sparse grid quadrature is available on https://github.com/muchip/SPQR.
} 
The computation of the solution $u_{0}$ of the unperturbed problem and the associated first and second order local shape derivaties are computed as introduced in Section 3 by employing 200 collocation points. The numerical solution of (23) for the particular samples is likewise determined by using the Dirichlet-to-Neumann map. A suitable Newton potential for (23) is given by the function

$$
N_{f}(\mathbf{x})=-\frac{1}{4}\left(x_{1}^{2}+x_{2}^{2}\right)
$$
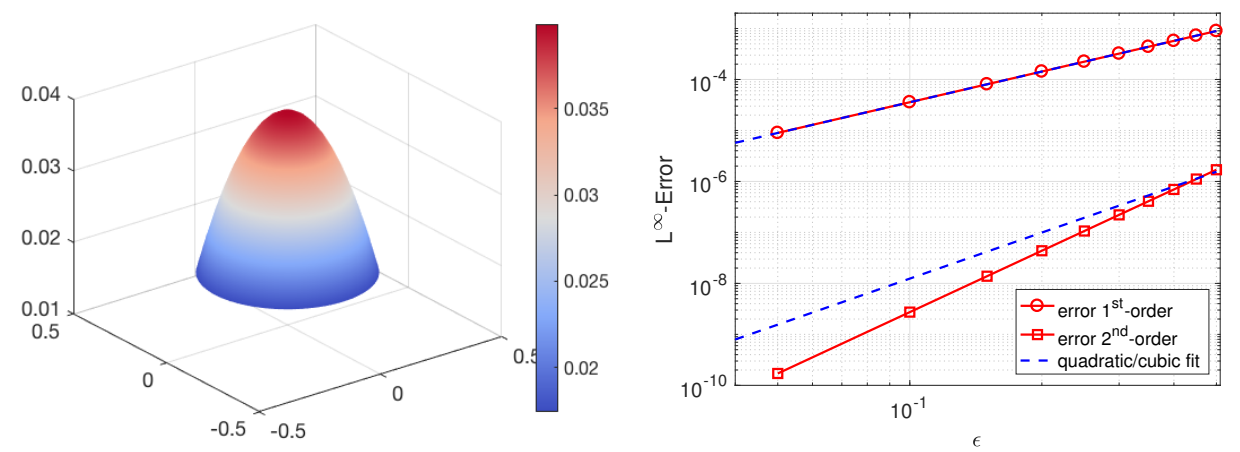

Figure 5: Visualization of the mean in case of $\varepsilon=1 / 2$ and the asymptotic behavior of the asymptotic expansion.

Figure 5 shows a visualization of the approximate mean in case of $\varepsilon=1 / 2$ on the left hand side. On the right hand side, the asymptotic behavior of the perturbation approach is presented in terms of the mean's $L^{\infty}$-error. It is evaluated on a grid similar to the one depicted in Figure 3 with about 2000 points. As a comparison, we also depicted the results which would be obtained by a first order shape Taylor expansion, resulting in the approximation $\mathbb{E}\left[u_{\varepsilon}\right](\mathbf{x})=u_{0}(\mathbf{x})+\mathcal{O}\left(\varepsilon^{2}\right)$. As it turns out, the first order perturbation approach perfectly provides the predicted quadratic rate, indicated by the dashed line. Here and in the sequel, the polynomial fits are obtained by a least squares fit of the coefficient of the respective monomial. For the second order perturbation approach, the cubic rate is not entirely met. In this concrete example, a quartic fit would reflect the error curve better.

As can be derived from (24), the first and second order perturbation approach coincide in case of the variance since the third order term just vanishes due to the symmetry of the random variables' distribution. A visualization of the approximate variance in case of $\varepsilon=1 / 2$ can be found on the left hand side of Figure 6 . Whereas, the asymptotic rate for the 

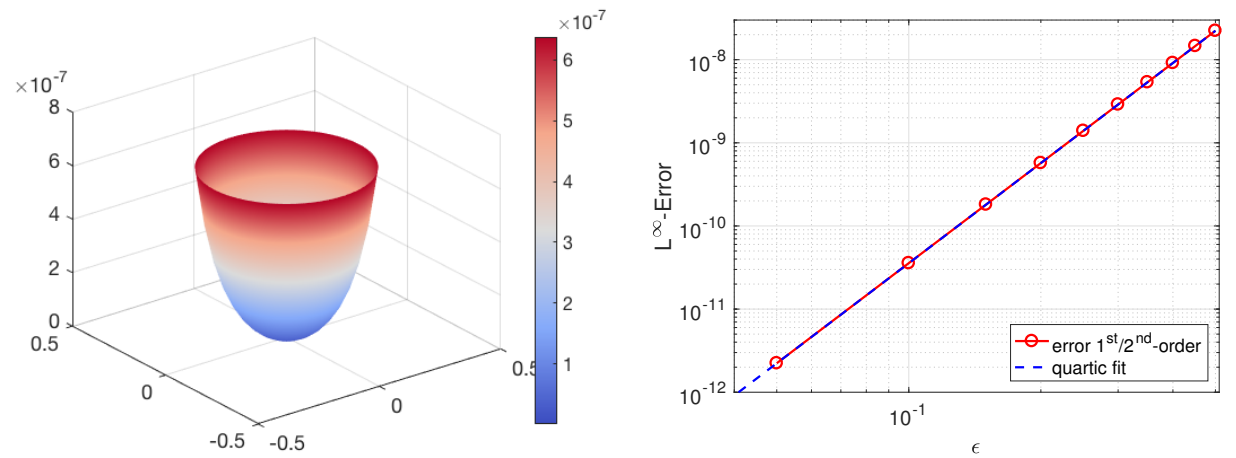

Figure 6: Visualization of the variance in case of $\varepsilon=1 / 2$ and the asymptotic behavior of the asymptotic expansion.

$L^{\infty}$-error of the variance is shown on the right hand side of Figure 6 . As can be seen, the error perfectly reflects the quartic fit.
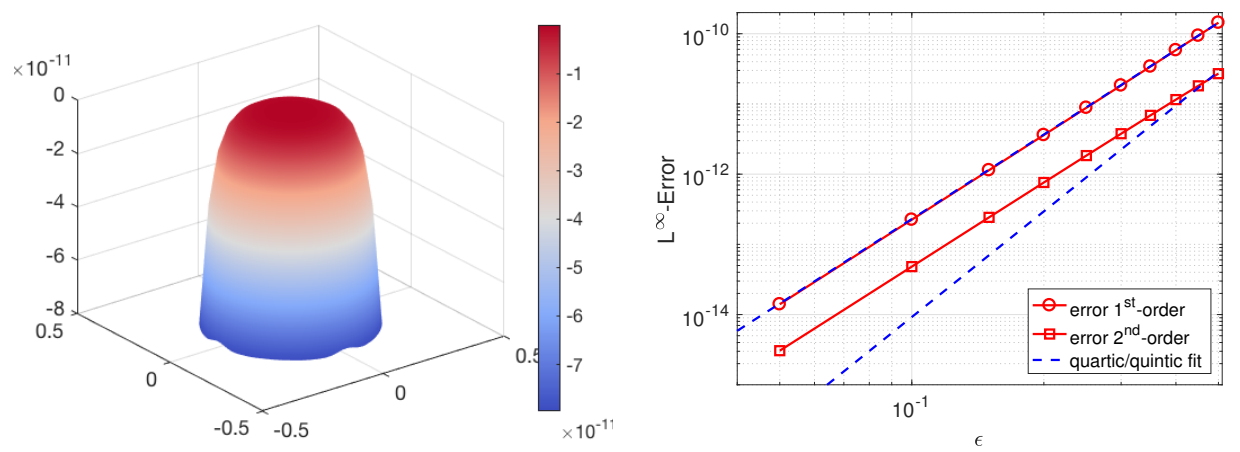

Figure 7: Visualization of the skewness in case of $\varepsilon=1 / 2$ and the asymptotic behavior of the asymptotic expansion.

The next moment to be considered is the random solution's skewness. On the left hand side of Figure 7, we find a visualization of the skewness in case of $\varepsilon=1 / 2$. The right hand side of Figure 7 contains the corresponding error for different sizes of $\varepsilon$. In difference to (11), the first order perturbation approach amounts to the expansion $\mathbb{M}_{3}\left[u_{\varepsilon}\right](\mathbf{x})=\mathcal{O}\left(\varepsilon^{4}\right)$. Indeed, we exactly observe this quartic rate in our computations. Compared to this, the expansion of the second order perturbation approach is up to one order of magnitude more precise. Nevertheless, the expected quintic rate is not entirely met. Due to the very small numbers that are involved 
in the corresponding computation, we assume that this effect is caused by cancellation.
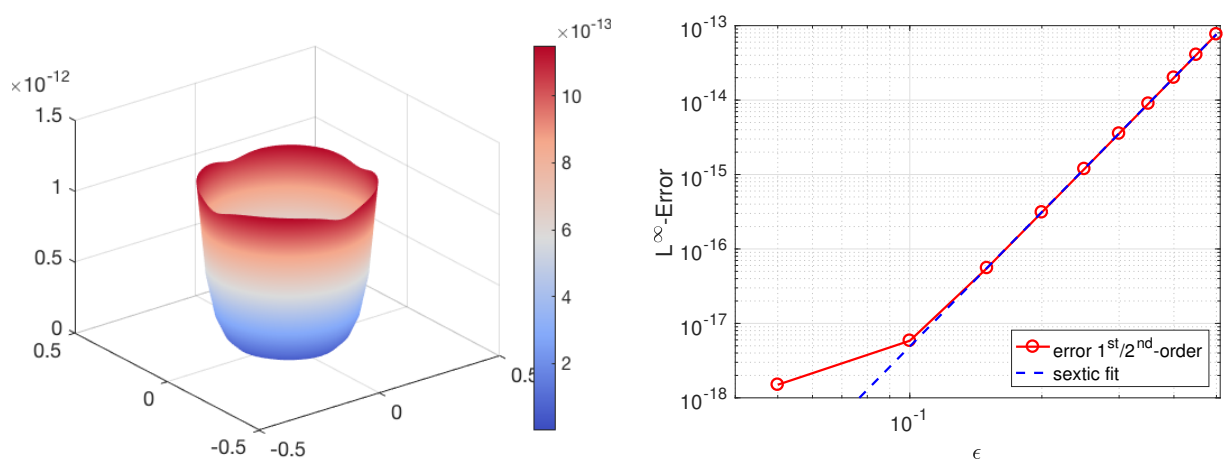

Figure 8: Visualization of the kutorsis in case of $\varepsilon=1 / 2$ and the asymptotic behavior of the asymptotic expansion.

Finally, we consider the random solution's kurtosis. It is depicted on the left hand side of Figure 8 for the choice $\varepsilon=1 / 2$. As in the case of the variance, the first and second order perturbation approach coincide due to the symmetry of the random variables. As can be seen in the right hand side of Figure 8, the asymptotic expansion's error exhibits the predicted sextic rate, except for the first value $\varepsilon=0.05$.

\section{Conclusion}

In the present article, we have applied the second order perturbation approach to boundary value problems that are defined with respect to random domains. To that end, we have derived asymptotic expansions for the expectation, the variance, the skewness and the kurtosis. In particular, we developed a boundary element method to efficiently compute these expansions. The application of the exponentially convergent Nyström approximation for this boundary integral equations keeps discretization errors at a low level. By numerical experiments, we have demonstrated that the second order perturbation approach is much more accurate than the first order perturbation approach. Especially when computing the expectation, we have observe rather a quartic rate than the expected cubic rate, whereas for the third moment, we still achieve an error that is up to an order of magnitude better than the error of the corresponding first order method. For the variance and the kurtosis, both approximations coincide. Therefore, the 
results corroborate our theoretical findings, except for the approximation of the skewness, where we assume that the reduced rate is caused by roundoff errors.

[1] I. Babuška and P. Chatzipantelidis. On solving elliptic stochastic partial differential equations. Comput. Meth. Appl. Mech. Engrg. 191 (2002) 4093-4122.

[2] H.-J. Bungartz and M. Griebel. Sparse grids. Acta Numer. 13 (2004) 147-269.

[3] R. Caflisch. Monte Carlo and quasi-Monte Carlo methods. Acta Numer. 7 (1998) 1-49.

[4] C. Canuto and T. Kozubek. A fictitious domain approach to the numerical solution of PDEs in stochastic domains. Numer. Math. 107 (2007) 257-293.

[5] J.E. Castrillon-Candas, F. Nobile, R.F. Tempone. Analytic regularity and collocation approximation for PDEs with random domain deformations. Comput. Math. Appl. 71(6) (2016) 1173-1197.

[6] M. Dambrine, H. Harbrecht, B. Puig. Computing quantities of interest for random domains with second order shape sensitivity analysis. ESAIM Math. Model. Numer. Anal. 49(5) (2015) 1285-1302.

[7] M. Delfour and J.-P. Zolesio. Shapes and Geometries. SIAM, Philadelphia, 2001.

[8] K. Eppler. Optimal shape design for elliptic equations via BIE-methods. Int. J. Appl. Math. Comput. Sci. 10 (2000) 487-516.

[9] K. Eppler. Boundary integral representations of second derivatives in shape optimization. Discuss. Math. Differ. Incl. Control Optim. 20 (2000) 63-78.

[10] L. C. Evans. Partial Differential Equations. Graduate Studies in Mathematics, American Mathematical Society, Providence, 2010.

[11] T. Gerstner and M. Griebel. Numerical integration using sparse grids. Numer. Algorithms 18 (1998) 209-232.

[12] J. Hadamard. Lectures on the Calculus of Variations. Gauthier-Villiars, Paris, 1910. 
[13] H. Harbrecht. On output functionals of boundary value problems on stochastic domains. Math. Meth. Appl. Sci. 33 (2010) 91-102.

[14] H. Harbrecht and J. Li. First order second moment analysis for stochastic interface problems based on low-rank approximation. ESAIM Math. Model. Numer. Anal. 47 (2013) 1533-1552.

[15] H. Harbrecht and M. Peters. Combination technique based second moment analysis for elliptic PDEs on random domains. In J. Garcke and D. Pflüger, editors, Sparse grids and applications, volume 109 of Lecture Notes in Computational Science and Engineering, pages 51-77, Springer International Publishing, Switzerland, 2016.

[16] H. Harbrecht, M. Peters, and M. Siebenmorgen. Combination technique based $k$-th moment analysis of elliptic problems with random diffusion. J. Comput. Phys. 252 (2013) 128-141.

[17] H. Harbrecht, R. Schneider, and C. Schwab. Sparse second moment analysis for elliptic problems in stochastic domains. Numer. Math. 109 (2008) 167-188.

[18] A. Henrot and M. Pierre Variation et Optimisation de Formes. Springer, Berlin-Heidelberg, 2005.

[19] M. Jung and O. Steinbach. A finite element-boundary element algorithm for inhomogeneous boundary value problems. Computing $\mathbf{6 8}$ (2002) 1-17.

[20] J.B. Keller. Stochastic Equations and Wave Propagation in Random Media. Bellman, 1964.

[21] M. Kleiber and T.D. Hien. The Stochastic Finite Element Method: Basic Perturbation Technique and Computer Implementation. Wiley, 1992.

[22] P.E. Kloeden and E. Platen. Numerical Solution of Stochastic Differential Equations. Springer-Verlag, 3rd ed., 1999.

[23] R. Kress. Linear Integral Equations. Vol. 82 of Applied Mathematical Sciences, Springer, New York, 2nd ed., 1999.

[24] M. Loève. Probability Theory. I+II. Volume 45 of Graduate Texts in Mathematics. Springer, Berlin-Heidelberg-New York, 4th ed., 1977. 
[25] P.S. Mohan, P.B. Nair, and A.J. Keane. Stochastic projection schemes for deterministic linear elliptic partial differential equations on random domains. Int. J. Numer. Meth. Engng. 85 (2011) 874-895.

[26] O. Pironneau. Optimal Shape Design for Elliptic Systems. Springer, New York, 1984.

[27] R. Potthast. Frechét-Differenzierbarkeit von Randintegraloperatoren und Randwertproblemen zur Helmholtzgleichung und zu den zeitharmonischen Maxwellgleichungen. PhD thesis, Georg-August-Universität Göttingen, 1994.

[28] P. Protter. Stochastic Integration and Differential Equations: A New Approach. 3rd edition, Springer, 1995.

[29] J. Sokolowski and J.-P. Zolesio. Introduction to Shape Optimization: Shape Sensitivity Analysis. Springer, 1992.

[30] D.M. Tartakovsky and D. Xiu. Numerical methods for differential equations in random domains. SIAM J. Sci. Comput. 28 (2006) 1167-1185. 